\title{
Mittag-Leffler Methods in Analysis
}

\author{
JORGE MUJICA
} 1993)

Dedicated to the memory of my teacher, Leopoldo Nachbin (1922-

ABSTRACT. In this survey we present two Mittag-Leffler lemmas and several applications to topics as varied as the $\bar{\partial}$-equation, Fréchet algebras, inductive limits of Banach spaces and quasi-normable Fréchet spaces.

\section{INTRODUCTION}

The classical Mittag-Leffler theorem asserts the existence of meromorphic functions with prescribed poles and singular parts. If the prescribed poles form a finite set, then it is clear that the sum of the corresponding singular parts is a function with the desired properties. But in the general case, that is when the prescribed poles form a sequence without accumulation points, then the corresponding series of singular parts is not necessarily convergent. But then, by means of suitable corrections of the terms of the series, so as to make it convergent, one obtains a function with the desired properties. 
Such a procedure has been so widely used in analysis, that has become known as Mittag-Leffler procedure. Several general results whose proofs follow such a procedure are often called Mittag-Leffler lemmas or Mittag-Leffler abstract theorems. In this survey we present two such lemmas and several applications to topics as varied as the $\bar{\partial}$ equation, Fréchet algebras, inductive limits of Banach spaces and quasi-normable Fréchet spaces.

This is the essential content of a lecture delivered at the IV Chilean Symposium of Mathematics, held at the Universidad de Santiago from September 27 to October 1, 1993. I wish to thank the organizers of the symposium for their kind invitation and financial support.

\section{MITTAG-LEFFLER LEMMAS}

Since we will be dealing with projective limits, we recall the definitions. Let $\left(X_{i}\right)_{i \in I}$ be a family of nonvoid sets, indexed by a directed set $I$. Suppose that for each pair of indices $i, j$ with $i \leq j$ there is a mapping $\xi_{i j}: X_{j} \rightarrow X_{i}$ such that $\xi_{i i}$ is the identity mapping on $X_{i}$ for every $i$ and $\xi_{i j} \circ \xi_{j k}=\xi_{i k}$ whenever $i \leq j \leq k$. Then the collection $\left(X_{i}, \xi_{i j}\right)$ is said to be a projective system, and the set

$$
X=\left\{\left(x_{i}\right) \in \prod_{i \in I} X_{i}: \xi_{i j}\left(x_{j}\right)=x_{i} \text { whenever } i \leq j\right\}
$$

is called the projective limit of the sets $X_{i}$ and is denoted by proj $X_{i}$. The canonical mapping $X \rightarrow X_{i}$ is denoted by $\xi_{i}$. If each $X_{i}$ is a topological space (resp. a group, a vector space, etc.) and each $\xi_{i j}$ is continuous (resp. a homomorphism, linear, etc.), then $X$ is a topological space (resp. a group, a vector space, etc.) as a subset of the product $\prod_{i \in I} X_{i}$ and each $\xi_{i}$ is continuous (resp. a homomorphism, linear, etc.).

The following lemma sharpens results of Arens [1, Theorem 2.4] and Esterle [6, Theorem 2.1].

1.1. Lemma. Let $X=\operatorname{proj}\left(X_{m}, d_{m}\right)$ be the projective limit of a sequence of complete metric spaces such that

$$
d_{m}\left(\xi_{m, m+1}(x), \xi_{m, m+1}(y)\right) \leq d_{m+1}(x, y) \text { for all } x, y \in X_{m+1} \text {. }
$$


Suppose that each $X_{m}$ contains a nonvoid set $T_{m}$ such that

$$
d_{m}\left(t, \xi_{m, m+1}\left(T_{m+1}\right)\right)<\varepsilon_{m} \text { for all } t \in T_{m}
$$

where $\varepsilon_{m}>0$ for every $m \in \mathbb{N}$ and $\sum_{m=1}^{\infty} \varepsilon_{m}<\infty$. Then the set

$$
B_{m}=\bigcap_{n=m}^{\infty} \bigcup_{t \in T_{n}} \xi_{n}^{-1}\left(B_{X_{n}}\left(t, \sum_{k=n}^{\infty} \varepsilon_{k}\right)\right)
$$

is nonvoid for every $m \in \mathbb{N}$ and

$$
d_{m}\left(t, \xi_{m}\left(B_{m}\right)\right) \leq \sum_{k=m}^{\infty} \varepsilon_{k} \text { for all } t \in T_{m} .
$$

Proof. Fix $m \in \mathbb{N}$ and $t_{m} \in T_{m}$. By repeated applications of (1.2) we can find $t_{n} \in T_{n}$ for every $n>m$ such that

$$
d_{n}\left(t_{n}, \xi_{n, n+1}\left(t_{n+1}\right)\right)<\xi_{n} \text { for every } n \geq m \text {. }
$$

We claim that $\left(\xi_{n p}\left(t_{p}\right)\right)_{p=n}^{\infty}$ is a Cauchy sequence in $X_{n}$ for every $n \geq m$. Indeed for $q \geq p \geq n \geq m$ we have that

$$
\begin{aligned}
& d_{n}\left(\xi_{n p}\left(t_{p}\right), \xi_{n q}\left(t_{q}\right)\right) \leq \sum_{k=p}^{q-1} d_{n}\left(\xi_{n k}\left(t_{k}\right), \xi_{n, k+1}\left(t_{k+1}\right)\right) \\
& \leq \sum_{k=p}^{q-1} d_{k}\left(t_{k}, \xi_{k, k+1}\left(t_{k+1}\right)\right)<\sum_{k=p}^{\infty} \varepsilon_{k} .
\end{aligned}
$$

Let $x_{n}=\lim _{p \rightarrow \infty} \xi_{n p}\left(t_{p}\right) \in X_{n}$ for every $n \geq m$. Since $\xi_{n p} \circ \xi_{p q}\left(t_{q}\right)=\xi_{n q}\left(t_{q}\right)$ for $q \geq p \geq n \geq m$, we see that $\xi_{n p}\left(x_{p}\right)=x_{n}$ for $p \geq n \geq m$. If we define $x_{n}=\xi_{n m}\left(x_{m}\right)$ for every $n<m$, we see that $x=\left(x_{n}\right)_{n=1}^{\infty}$ belongs to $X$. By taking $p=n$ and $q \rightarrow \infty$ in (1.5) we get that 


$$
d_{n}\left(t_{n}, x_{n}\right) \leq \sum_{k=n}^{\infty} \varepsilon_{k} \text { for every } n \geq m .
$$

This shows that $x \in B_{m}$ and $d_{m}\left(t_{m}, \xi_{m}(x)\right) \leq \sum_{k=m}^{\infty} \varepsilon_{k}$, as we wanted.

1.2 Corollary (Esterle [6]). Let $X=\operatorname{proj}\left(X_{m}, d_{m}\right)$ be the projective limit of a sequence of complete metric spaces such that

$$
d_{m}\left(\xi_{m, m+1}(x), \xi_{m, m+1}(y)\right) \leq d_{m+1}(x, y) \text { for all } x, y \in X_{m+1} .
$$

Suppose that

$$
d_{m}\left(x, \xi_{m, m+1}\left(X_{m+1}\right)\right)<\varepsilon_{m} \text { for all } x \in X_{m} \text { and } m \in \mathbf{N}
$$

where $\varepsilon_{m}>0$ for every $m \in \mathbb{N}$ and $\sum_{m=1}^{\infty} \varepsilon_{m}<\infty$. Then $X$ is nonvoid and

$$
d_{m}\left(x, \xi_{m}(X)\right) \leq \sum_{k=m}^{\infty} \varepsilon_{k} \text { for all } x \in X_{m} \text { and } m \in \mathbb{N}
$$

1.3 Corollary. Let $X=$ proj $X_{m}$ be the projective limit of a sequence of complete metric spaces. Suppose that each $X_{m}$ contains a nonvoid set $T_{m}$ such that $T_{m} \subseteq \overline{\xi_{m, m+1}\left(T_{m+1}\right)}$ for every $m \in \mathbb{N}$. Then $X$ is nonvoid and $T_{m} \subset \overline{\xi_{m}(X)}$ for every $m \in \mathbb{N}$. define

Proof. Let $\delta_{m}$ denote the metric on $X_{m}$ for every $m \in \mathbf{N}$ and

$$
d_{m}(x, y)=\max _{1 \leq n \leq m} \delta_{n}\left(\xi_{n m}(x), \xi_{n m}(y)\right) \text { for all } x, y \in X_{m}
$$

Since each $\xi_{n m}$ is continuous, $d_{m}$ and $\delta_{m}$ define the same topology on $X_{m}$. And since $\left(X_{m}, \delta_{m}\right)$ is complete, one can readily verify that $\left(X_{m}, d_{m}\right)$ is complete. Since $d_{m}$ satisfies condition (1.1) in Lemma 1.1, the desired conclusion follows.

The next result, due to Arens [1, Theorem 2.4], is probably the first Mittag-Leffler lemma. 
1.4 Corollary (Arens [1]). Let $X=$ proj $X_{m}$ be the projective limit of a sequence of complete metric spaces. If each $\xi_{m, m+1}: X_{m+1} \rightarrow X_{m}$ has a dense range, then $X$ is nonvoid and each $\xi_{m}: X \rightarrow X_{m}$ has a dense range.

The next lemma summarizes results of Palamodov [19, p. 215, Proposition 11], Kornatsu [12, Lemma 1.3], Bierstedt et al. [3, Lemma 2.8] and Galbis [8]. We shall derive this lemma from Corollary 1.3.

1.5 Lemma. Let $X=\operatorname{proj} X_{m}, Y=\operatorname{proj} Y_{m}$ and $Z=\operatorname{proj} Z_{m}$ be the projective limits of three sequences of abelian groups. Suppose that for every $m \in \mathbb{N}$ there are homomorphisms $\varphi_{m}: X_{m} \rightarrow Y_{m}$ and $\psi_{m}: Y_{m} \rightarrow Z_{m}$ such that the following diagram is commutative and each row is exact.

$$
\begin{array}{lllll}
X_{m} & \stackrel{\varphi_{m}}{\longrightarrow} & Y_{m} & \stackrel{\psi_{m}}{\longrightarrow} & Z_{m} \\
\uparrow \xi_{m, m+1} & & \uparrow \eta_{m, m+1} & & \uparrow \zeta_{m, m+1} \\
X_{m+1} & \stackrel{\varphi_{m+1}}{\longrightarrow} & Y_{m+1} & \stackrel{\psi_{m+1}}{\longrightarrow} & Z_{m+1}
\end{array}
$$

Let $\varphi: X \rightarrow Y$ and $\psi: Y \rightarrow Z$ be the unique homomorphisms such that the following diagram is commutative and each row is exact.

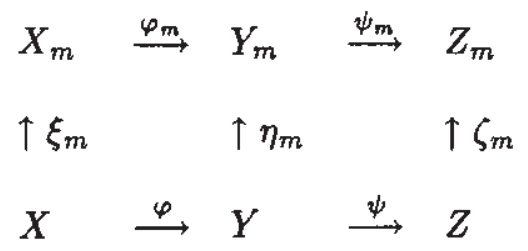

(a) If each $\varphi_{m}$ is injective, then $\varphi$ is injective.

(b) Suppose that $X=\operatorname{proj} X_{m}$ is actually a projective limit of complete, abelian, metric groups. Suppose in addition that

$$
\xi_{m, m+1}\left(X_{m+1}\right) \subset \overline{\xi_{m, m+2}\left(X_{m+2}\right)} \text { for every } m \in \mathbb{N}
$$


Under these conditions, if $\zeta_{m}(Z) \subset \psi_{m}\left(Y_{m}\right)$ for every $m \in \mathbb{N}$, then $\psi$ is surjective.

Proof. If $\varphi(x)=\left(\varphi_{m}\left(x_{m}\right)\right)$ for every $x=\left(x_{m}\right) \in X$, and $\psi(y)=$ $\left(\psi_{m}\left(y_{m}\right)\right)$ for every $y=\left(y_{m}\right) \in Y$, then the only nontrivial assertion in the lemma is the surjectivity of $\psi$. Let $z=\left(z_{m}\right) \in Z$. Since $\zeta_{m}(Z) \subset$ $\psi_{m}\left(Y_{m}\right)$, for each $m \in \mathbb{N}$ there exists $y_{m} \in Y_{m}$ such that $\psi_{m}\left(y_{m}\right)=z_{m}$. Then $\left(y_{m}\right) \in \Pi Y_{m}$, but there is no guarantee that $\left(y_{m}\right) \in Y$. The idea is to find a sequence of corrections $x_{m}$, with $x_{m} \in X_{m}$ for every $m \in \mathbb{N}$, such that $\left(y_{m}-\varphi_{m}\left(x_{m}\right)\right) \in Y$. Since

$$
\psi_{m}\left(y_{m}-\varphi_{m}\left(x_{m}\right)\right)=\psi_{m}\left(y_{m}\right)=z_{m}
$$

for every $m \in \mathbf{N}$, this will complete the proof. Thus we want to find $\left(x_{m}\right) \in \Pi X_{m}$ such that

$$
\eta_{m, m+1}\left(y_{m+1}-\varphi_{m+1}\left(x_{m+1}\right)\right)=y_{m}-\varphi_{m}\left(x_{m}\right)
$$

that is

$$
\begin{aligned}
\eta_{m, m+1}\left(y_{m+1}\right)-y_{m} & =\eta_{m, m+1} \circ \varphi_{m+1}\left(x_{m+1}\right)-\varphi_{m}\left(x_{m}\right)= \\
& =\varphi_{m}\left(\xi_{m, m+1}\left(x_{m+1}\right)-x_{m}\right)
\end{aligned}
$$

for every $m \in \mathbb{N}$. Now since $\left(z_{m}\right) \in Z$ we have that

$$
\begin{aligned}
\psi_{m}\left(\eta_{m, m+1}\left(y_{m+1}\right)-y_{m}\right) & =\zeta_{m, m+1} \circ \psi_{m+1}\left(y_{m+1}\right)-\psi_{m}\left(y_{m}\right)= \\
& =\zeta_{m, m+1}\left(z_{m+1}\right)-z_{m}=0
\end{aligned}
$$

and therefore $\eta_{m, m+1}\left(y_{m+1}\right)-y_{m} \in \psi_{m}^{-1}(0)=\varphi_{m}\left(X_{m}\right)$. Thus for each $m \in \mathbb{N}$ there exists $a_{m} \in X_{m}$ such that

$$
\varphi_{m}\left(a_{m}\right)=\eta_{m, m+1}\left(y_{m+1}\right)-y_{m}
$$


Comparing (1.7) and (1.8) we see that to complete the proof it suffices to find $\left(x_{m}\right) \in \mathbb{I I} X_{m}$ such that

$$
\varphi_{m}\left(a_{m}\right)=\varphi_{m}\left(\xi_{m, m+1}\left(x_{m+1}\right)-x_{m}\right)
$$

for every $m \in \mathbb{N}$. Now let $\tilde{\xi}_{m, m+1}: X_{m+1} \rightarrow X_{m}$ de defined by

$$
\tilde{\xi}_{m, m+1}(x)=\xi_{m, m+1}(x)-a_{m}
$$

for every $x \in X_{m+1}$, and let $\tilde{X}$ denote the projective limit of the complete metric spaces $X_{m}$ with respect to the linking mappings $\bar{\xi}_{m, m+1}$ : $X_{m+1} \rightarrow X_{m}$. If we use (1.6), then a straightforward verification shows that

$$
\tilde{\xi}_{m, m+1}\left(X_{m+1}\right) \subset \overline{\tilde{\xi}_{m, m+2}\left(X_{m+2}\right)} \text { for every } m \in \mathbb{N} \text {. }
$$

If we set $T_{m}=\tilde{\xi}_{m, m+1}\left(X_{m+1}\right)$ for every $m \in \mathbb{N}$, then Corollary 1.3 applies and guarantees that $\tilde{X}$ is nonvoid. If $\left(x_{m}\right) \in \bar{X}$, then $\bar{\xi}_{m, m+1}\left(x_{m+1}\right)$ $=x_{m}$, that is $\xi_{m, m+1}\left(x_{m+1}\right)-a_{m}=x_{m}$ for every $m \in \mathbb{N}$. This achieves (1.9) and completes the proof.

Let us mention that Petzsche [20] derives the classical Mittag-Leffler theorem from Lemma 1.5, whereas Esterle [6] derives the Baire category theorem and the classical Mittag-Leffler theorem from Corollary 1.4.

1.6. Remark. In all of the preceding lemmas we required that $X=$ proj $X_{m}$ be the projective limit of a sequence of complete metric spaces. An examination of the proofs shows that the conclusions remain true if $X=$ proj $X_{m}$ is the projective limit of a sequence of pseudometric spaces with the property that whenever $\left(a_{p}\right)$ is a Cauchy sequence in $X_{m+1}$, then the sequence $\left(\xi_{m, m+1}\left(a_{p}\right)\right)$ has a unique limit in $X_{m}$.

\section{THE $\tilde{\partial}$ EQUATION ON POLYNOMIALLY CONVEX DO- MAINS}

If $U$ is an open set in $\mathbb{C}^{\mathrm{n}}$, then $\mathcal{C}_{p q}^{\infty}(U)$ denotes the vector space of all $C^{\infty}$ differential forms of type $(p, q)$ on $U$. If $K$ is a compact set in $\mathbb{C}^{\mathrm{n}}$, then $\mathcal{C}_{p q}^{\infty}(K)$ denotes the vector space of all germs of $C^{\infty}$ differential forms of type $(p, q)$ around $K$. 
The solution of the $\tilde{\partial}$ equation around polynomially convex compact sets is essentially due to Oka [18] (see Hörmander [11, Lemma 2.7.4 and Theorem 2.7.6]). And then a standard Mittag-Leffier procedure extends the solution to the case of polinomially convex open sets (see Hörmander [11, Theorem 2.7.8]). Let us see how this result follows from Lemma 1.5.

2.1. Theorem (Oka [18], Hörmander [11]). Let $U$ be a polynomially convex open set in $\mathbb{C}^{\mathbf{n}}$. Then for each $g \in \mathcal{C}_{p, q+1}^{\infty}(U)$ with $\bar{\partial} g=0$, there exists $f \in \mathcal{C}_{p q}^{\infty}(U)$ such that $\bar{\partial} f=g$.

Proof. Let $\left(K_{m}\right)$ be a sequence of polynomially convex compact sets such that $U=\cup_{m=1}^{\infty} K_{m}$ and $K_{m} \subset$ int $K_{m+1}$ for every $m \in \mathbb{N}$.

(a) Consider first the case $q \geq 1$. If $\mathcal{F}_{p q}^{\infty}(U)$ (resp. $\mathcal{F}_{p q}^{\infty}(K)$ ) denotes the subspace of all $f \in \mathcal{C}_{p q}^{\infty}(U)$ (resp. $\mathcal{C}_{p q}^{\infty}(K)$ ) with $\bar{\partial} f=0$, then we have the following commutative diagram of vector spaces and linear mappings.

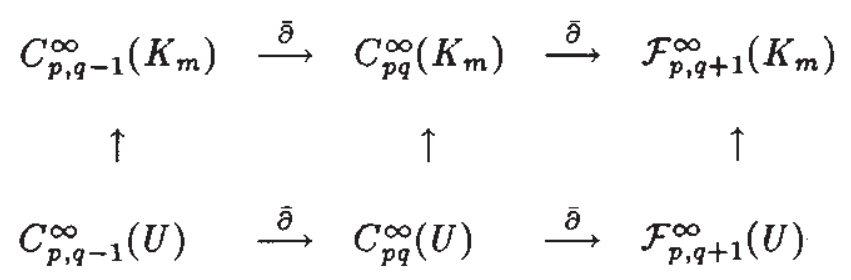

Then the mappings $\bar{\partial}: \mathcal{C}_{p q}^{\infty}\left(K_{m}\right) \rightarrow \mathcal{F}_{p, q+1}^{\infty}\left(K_{m}\right)$ and $\mathcal{C}_{p, q-1}^{\infty}(U) \rightarrow$ $\mathcal{C}_{p, q-1}^{\infty}\left(K_{m}\right)$ are surjective. If we endow each of the spaces $\mathcal{C}_{p, q-1}^{\infty}\left(K_{m}\right)$ with the discrete metric, then Lemma 1.5 applies and guarantees that the mapping $\bar{\partial}: \mathcal{C}_{p q}^{\infty}(U) \rightarrow \mathcal{F}_{p, q+1}^{\infty}(U)$ is surjective.

(b) Consider next the case $q=0$. Recall that if $f \in \mathcal{C}_{p 0}^{\infty}(U)$, then $\bar{\partial} f=0$ if and only if $f$ is a holomorphic mapping on $U$ with values in $\mathcal{L}^{a}\left({ }^{p} \mathbb{C}^{n}\right.$ ), the Banach space of all alternating $p$-linear forms on $\mathbb{C}^{n}$ (see Mujica [16, Proposition 21.6]). Then we have the following commutative diagram of vector spaces and linear mappings. 


$$
\begin{aligned}
& \mathcal{H}\left(K_{m} ; \mathcal{L}^{a}\left(\mathfrak{P}^{\mathrm{n}}\right)\right) \quad \hookrightarrow \quad C_{p 0}^{\infty}\left(K_{m}\right) \stackrel{\check{\partial}}{\longrightarrow} \mathcal{F}_{p 1}^{\infty}\left(K_{m}\right) \\
& \text {. } \uparrow \uparrow \uparrow \uparrow \\
& \mathcal{H}\left(U ; \mathcal{L}^{a}\left(\boldsymbol{P}^{\mathrm{n}}\right)\right) \quad \hookrightarrow \quad C_{p 0}^{\infty}(U) \stackrel{\Xi}{\longrightarrow} \mathcal{F}_{p 1}^{\infty}(U)
\end{aligned}
$$

Then the mapping $\bar{\partial}: \mathcal{C}_{p 0}^{\infty}\left(K_{m}\right) \rightarrow \mathcal{F}_{p 1}^{\infty}\left(K_{m}\right)$ is surjective for every $m \in \mathbb{N}$. If $\mathcal{H}\left(K_{m} ; \mathcal{L}^{a}\left({ }^{p} \mathbb{C}^{n}\right)\right)$ is endowed with the seminorm of the supremum on $K_{m}$, then a theorem of Oka (see Hörmander [11, Theorem 2.7.7]) implies that the mapping $\mathcal{H}\left(U ; \mathcal{L}^{a}\left({ }^{\mathrm{n}}\right)\right) \rightarrow \mathcal{H}\left(\mathrm{K}_{\mathrm{m}} ; \mathcal{L}^{\mathrm{a}}\left({ }^{\mathrm{n}} \mathbb{C}^{\mathrm{n}}\right)\right)$ has a dense range. By Lemma 1.5 and Remark 1.6 the mapping $\vec{\partial}: \mathcal{C}_{p 0}^{\infty}(U) \rightarrow$ $\mathcal{F}_{p 1}^{\infty}(U)$ is surjective.

\section{FRECHET ALGEBRAS}

If $A$ is a commutative Fréchet algebra, then $S(A)$ denotes the spectrum of $A$, that is the set of all continuous nonzero homomorphisms $\varphi: A \rightarrow \mathbb{C}$. All Fréchet algebras are assumed to have an identity.

3.1. Theorem. (Brooks [5]). Let A be a commutative Fréchet algebra. Let $\left(a_{j}\right)$ be a sequence in $A$ such that

$$
\bigcap_{j=1}^{\infty}\left\{\varphi \in S(A): \varphi\left(a_{j}\right)=0\right\}=\phi
$$

Then there is a sequence $\left(x_{j}\right)$ in $A$ such that $\sum_{j=1}^{\infty} a_{j} x_{j}=1$.

Theorem 3.1 is due to Brooks [5, Theorem 2.2] in the case of infinite sequences, and to Arens [1, Theorem 4.2] in the case of finite sequences. Arens obtained the result with the aid of Corollary 1.4 and the following lemma.

3.2. Lemma. (Arens [1]). Let $A$ and $B$ be two commutative Banach algebras and let $\pi: A \rightarrow B$ be a homomorphism with a dense 
range. Let $a_{1}, \ldots, a_{p} \in A$ such that $A=a_{1} A+\cdots+a_{p} A$. Then, given $\varepsilon>0$ and $y_{1}, \ldots, y_{p} \in B$ such that $\pi\left(a_{1}\right) y_{1}+\cdots+\pi\left(a_{p}\right) y_{p}=1$, there are $x_{1}, \ldots, x_{p} \in A$ such that $a_{1} x_{1}+\cdots+a_{p} x_{p}=1$ and $\left\|\pi\left(x_{j}\right)-y_{j}\right\| \leq \varepsilon$ for $j=1, \ldots, p$.

In the case of infinite sequences, Corollary 1.4 is not strong enough to prove Theorem 3.1, and Brooks gave a direct, rather cumbersome proof (see also Goldmann $[9$, p. 136]). We now prove Theorem 3.1 with the aid of Corollary 1.3 .

Proof of Theorem 3.1. By a result of Michael [14, Theorem 5.1], $A$ can be represented as the projective limit of a sequence of commutative Banach algebras $A_{m}$, where each homomorphism $\pi_{m}: A \rightarrow A_{m}$ has a dense range. It follows from (3.1) that

$$
\bigcap_{j=1}^{\infty}\left\{\varphi \in S\left(A_{m}\right): \varphi \circ \pi_{m}\left(a_{j}\right)=0\right\}=\phi
$$

for every $m \in \mathbb{N}$. Since the sets $S\left(A_{m}\right)$ are all compact, we can find an increasing sequence $\left(p_{m}\right)$ in $\mathbb{N}$ such that

$$
\bigcap_{j=1}^{p_{m}}\left\{\varphi \in S\left(A_{m}\right): \varphi \circ \pi_{m}\left(a_{j}\right)=0\right\}=\phi
$$

for every $m \in \mathbb{N}$. Since each $A_{m}$ is a Banach algebra, we can find $y_{1}, \ldots, y_{p_{m}} \in A_{m}$ such that $\sum_{j=1}^{p_{m}} \pi_{m}\left(a_{j}\right) y_{j}=1$. We will now show the existence of a sequence $\left(x_{j}\right)$ in $A$ such that $\sum_{j=1}^{\infty}\left\|\pi_{m}\left(a_{j}\right)\right\|\left\|_{m}\right\| \pi_{m}\left(x_{j}\right) \|_{m}<$ $\infty$ for every $m \in \mathbb{N}$ and $\sum_{j=1}^{\infty} a_{j} x_{j}=1$. This will complete the proof. For each $m \in \mathbb{N}$ consider the Banach space

$$
E_{m}=\left\{\left(x_{j}\right) \in A_{m}^{\mathrm{N}}:\left\|\left(x_{j}\right)\right\|_{m}:=\sum_{j=1}^{\infty}\left\|\pi_{m}\left(a_{j}\right)\right\|_{m}\left\|x_{j}\right\|_{m}<\infty\right\}
$$


and its subsets

$$
X_{m}=\left\{\left(x_{j}\right) \in E_{m}: \sum_{j=1}^{\infty} \pi_{m}\left(a_{j}\right) x_{j}=1\right\}
$$

and

$$
T_{m}^{*}=\left\{\left(x_{j}\right) \in X_{m}: x_{j}=0 \text { for every } j>p_{m}\right\} .
$$

Thus $X_{m}$ is a complete metric space, as a closed subset of $E_{m}$. Consider also the Fréchet space

$$
\begin{aligned}
E & =\left\{\left(x_{j}\right) \in A^{\mathbf{N}}:\left\|\left(x_{j}\right)\right\|_{m}:=\right. \\
& \left.=\sum_{j=1}^{\infty}\left\|\pi_{m}\left(a_{j}\right)\right\|_{n}\left\|\pi_{m}\left(x_{j}\right)\right\|_{m}<\infty \text { for all } m \in \mathbb{N}\right\}
\end{aligned}
$$

and its subset

$$
X=\left\{\left(x_{j}\right) \in E: \sum_{j=1}^{\infty} a_{j} x_{j}=1\right\}
$$

Then $E=\operatorname{proj} E_{m}$ and $X=\operatorname{proj} X_{m}$. If $\xi_{m, m+1}: X_{m+1} \rightarrow X_{m}$ denotes the natural mapping, then it follows from Lemma 3.2 that $T_{m} \subset$ $\overline{\xi_{m, m+1}\left(T_{m+1}\right)}$ for every $m \in \mathbb{N}$. By Corollary $1.3 X$ is nonvoid.

In a similar manner we can use Corollary 1.3 to prove another result of Brooks, namely [5, Theorem 2.4].

\section{INDUCTIVE LIMITS OF A BANACH SPACES}

We recall that if $F$ is a Fréchet space, then the inductive dual $F_{i}^{\prime}$ of $F$ is the inductive limit of the Banach spaces $\left(F^{\prime}\right)_{V_{m}^{0}}$, where $\left(V_{m}\right)$ is any basis of convex, balanced, 0 -neighborhoods in $F$. It follows from the work of Grothendieck (see [10, Théoréme 6]) that $F_{i}^{\prime}$ is always complete. We refer to Bierstedt's survey [2] for information on the inductive dual.

Let $E=$ ind $E_{m}$ be the inductive limit of an increasing sequence of Banach spaces. In [15, Theorem 1] we proved that if there is a Hausdorff locally convex topology $\tau$ on $E$ such that the closed unit ball of each $E_{m}$ is $\tau$-compact, then $E$ is topologically isomorphic to $F_{i}^{\prime}$ for a suitable Fréchet space $F$. In particular $E$ is complete. 
When trying to apply this theorem in concrete situations, it is natural to seek for a Hausdorff locally convex topology $\tau_{m}$ on each $E_{m}$ such that:

(a) the inclusion mapping $\left(E_{m}, \tau_{m}\right) \hookrightarrow\left(E_{m+1}, \tau_{m+1}\right)$ is continuous;

(b) the closed unit ball of $E_{m}$ is $\tau_{m}$-compact.

If the inductive limit $(E, \tau):=$ ind $\left(E_{m}, \tau_{m}\right)$ is Hausdorff, then the preceding theorem directly applies. But in certain situations it may be difficult to prove that $(E, \tau)$ is Hausdorff. Hence the following variant of the preceding theorem is sometimes more useful.

4.1. Theorem. Let $E=$ ind $E_{m}$ be the inductive limit of an increasing sequence of Banach spaces. Suppose that for each $m \in \mathbb{N}$ there is a Hausdorff locally convex topology $\tau_{m}$ on $E_{m}$ such that:

(a) the inclusion mapping $\left(E_{m}, \tau_{m}\right) \hookrightarrow\left(E_{m+1}, \tau_{m+1}\right)$ is continuous;

(b) the closed unit ball $B_{m}$ of $E_{m}$ is $\tau_{m}$-compact.

If we set

$F=\left\{\varphi \in E^{\prime}: \varphi \mid B_{m}\right.$ is $\tau_{m}-$ continuous for every $\left.m \in \mathbb{N}\right\}$,

then $F$ is a Fréchet space for the topology of uniform convergence on each $B_{m}$, and $E$ is topologically isomorphic to $F_{i}^{\prime}$. In particular $E$ is Hausdorff, regular and complete.

Proof. If we set

$$
F_{m}=\left\{\varphi \in E_{m}^{\prime}: \varphi \mid B_{m} \text { is } \tau_{m} \text { - continuous }\right\}
$$

then $F_{m}$ is a Banach space for the norm $\|\varphi\|=\sup |\varphi|$, and $F$ can be canonically identified with proj $F_{m}$. Let $R_{m}: \stackrel{B_{m}}{F} \rightarrow F_{m}$ and $R_{m n}$ : $F_{n} \rightarrow F_{m}(m \leq n)$ denote the restriction mappings. Let $J: E \rightarrow F^{\prime}$ and $J_{m}: E_{m} \rightarrow F_{m}^{\prime}$ denote the evaluation mappings. By a result of Waelbroeck [21] and $\mathrm{Ng}$ [17], $J_{m}$ is an isometric isomorphism for every $m \in \mathbb{N}$. Since $F=\operatorname{proj} F_{m}$, it is clear that $J$ is surjective, but it is far from clear that $J$ is injective. We shall prove that $F$ is indeed injective. 
Since $F_{i}^{j}=$ ind $F_{m}^{\prime}$, it will follow that $J: E \rightarrow F_{i}^{\prime}$ is a topological isomorphism. Now since the following diagram is commutative,

$$
\begin{array}{rllll} 
& E_{m} & \hookrightarrow & E_{m+1} & \\
J_{m} & \downarrow & & \downarrow & J_{m+1} \\
& & & & \\
& F_{m}^{\prime} & \stackrel{R_{m, m+1}^{\prime}}{\longrightarrow} & F_{m+1}^{\prime} &
\end{array}
$$

we see that the dual mapping $R_{m, m+1}^{\prime}: F_{m}^{\prime} \rightarrow F_{m+1}^{\prime}$ is injective. By the Hahn-Banach theorem the mapping $R_{m, m+1}: F_{m+1} \rightarrow F_{m}$ has a dense range for every $m \in \mathbb{N}$. By Corollary 1.4 the mapping $R_{m}: F \rightarrow F_{m}$ has a dense range as well, and hence the dual mapping $R_{m}^{\prime}: F_{m}^{\prime} \rightarrow F^{\prime}$ is injective. Since the following diagram is commutative,

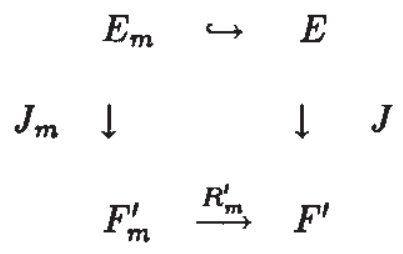

we see that the mapping $J: E \rightarrow F^{\prime}$ is injective, as we wanted.

The following corollary improves a result of Floret [7, Corollary 2].

4.2. Corollary. Let $E=$ ind $G_{m}^{\prime}$ be the inductive limit of an increasing sequence of duals $G_{m}^{\prime}$ of Banach spaces $G_{m}$ such that the inclusion mappings $G_{m}^{\prime} \hookrightarrow G_{m+1}^{\prime}$ are dual mappings. If $G:=\operatorname{proj} G_{m}$, then $E$ is topologically isomorphic to $G_{i}^{\prime}$. In particular $E$ is Hausdorff, regular and complete.

Proof. Apply Theorem 4.1 with $\tau_{m}=\sigma\left(G_{m}^{\prime}, G_{m}\right)$ for every $m \in \mathbf{N}$. It follows from Grothendieck's characterization of the completion that each $F_{m}$ coincides with $G_{m}$, and hence $F$ coincides with $G$.

I obtained these results in 1986, but did not publish them. I communicated the results to Klaus Bierstedt, who quoted them without proofs in his survey on inductive limits (see [2, Theorem 3.15]). 


\section{QUASI-NORMABLE FRECHET SPACES}

Quasi-normable spaces were introduced by Grothendieck [10]. A Fréchet space $E$ is said to be quasi-normable if it has a decreasing basis of closed, convex, balanced 0-neighborhoods $U_{m}$ such that for each $m \in \mathbf{N}$ and each $\varepsilon>0$ there is a bounded set $B \subset E$ such that

$$
U_{m+1} \subset \varepsilon U_{m}+B
$$

The following theorem is part of a result of Bonet [4].

5.1. Theorem. (Bonet [4]). A Fréchet space $E$ is quasi-normable if and only if has a decreasing basis of closed, convex, balanced 0 -neighborhoods $U_{m}$ such that for each $m \in \mathbf{N}$, each $\varepsilon>0$ and each $n>m$ there exists $\lambda>0$ such that

$$
U_{m+1} \subset \varepsilon U_{m}+\lambda U_{n}
$$

Proof. If $B$ is a bounded subset of $E$, then $B \subset \bigcap_{n=1}^{\infty} \lambda_{n} U_{n}$ for suitable $\lambda_{n}>0$, and hence it is clear that (5.1) implies (5.2). Bonet [4] gave two proofs of the reverse implication. One proof is based on a result of Meise and Vogt [13, Theorem 7], and the other one is based on a Mittag-Leffler procedure. We now derive this implication from Lemma 1.1. Fix $m \in \mathbb{N}$ and $\varepsilon>0$. By using (5.2) and induction we can easily find a sequence $\left(\mu_{n}\right)_{n=m+1}^{\infty}$, with $\mu_{m+1}=1$ and $\mu_{n}>0$ for all $n>m$ such that

$$
\mu_{n+1} U_{n+1} \subset \frac{\varepsilon}{2^{n}} U_{n}+\mu_{n+2} U_{n+2} \text { for all } n \geq m .
$$

Without loss of generality we way assume that $E=\operatorname{proj} E_{n}$ is the projective limit of a sequence of Banach spaces, and $U_{n}=\xi_{n}^{-1}\left(V_{n}\right)$ is the inverse image of the closed unit ball of $E_{n}$. Set $T_{n}=\xi_{n}\left(\mu_{n+1} U_{n+1}\right)$ for every $n \geq m$. Since we may assume that the natural mapping $\xi_{n, n+1}$ : $E_{n+1} \rightarrow E_{n}$ has norm not greater than one, condition (1.1) in Lemma 
1.1 is satisfied. And (5.3) guarantees that condition (1.2) is satisfied too with $\varepsilon_{n}=\varepsilon / 2^{n}$. Then Lemma 1.1 implies that

$$
T_{m} \subset \xi_{m}\left(B_{m}\right)+\varepsilon V_{m},
$$

where

$$
B_{m} \subset \bigcap_{n=m}^{\infty}\left(\mu_{n+1} U_{n+1}+\varepsilon U_{n}\right) \subset \bigcap_{n=m}^{\infty}\left(\mu_{n+1}+\varepsilon\right) U_{n} .
$$

Thus $B_{m}$ is a bounded subset of $E$ and $U_{m+1} \subset B_{m}+\varepsilon U_{m}$, as we wanted.

\section{References}

[1] Arens, R., Dense inverse limit rings, Michigan Math. J. 5 (1958), $169-182$.

[2] Bierstedt, K.D., An introduction to locally convex inductive limits. In: Functional Analysis and its Applications, edited by H. Hogbe-Nlend, World Scientific, Singapore, 1988, pp. 35-133.

[3] Bierstedt, K.D., Gramsch, B. and Meise, R., Approximationseigenschaft, Lifting und Kohomologie bei lokalkonvexen produktgarben, Manuscripta Math. 19 (1976), 319-364.

[4] Bonet, J., A question of Valdivia on quasinormable Fréchet spaces, Canad. Math. Bull. 34 (1991), 301-304.

[5] Brooks, R.M., Partitions of unity in F-algebras, Math. Ann. 177 (1968), 265-272.

[6] Esterle, J., Mittag-Leffler methods in the theory of Banach algebras and a new approach to Michael's problem. In: Proceedings of the Conference on Banach Algebras and Several Complex Variables, edited by F. Greenleaf and D. Gulick, Contemporary Mathematics 32, American Mathematical Society, Providence, Rhode Island, 1984, pp. 107-129.

[7] Floret, K., On bounded sets in inductive limits of normed spaces, Proc. Amer. Math. Soc. 75 (1979), 221-225.

[8] Galbis, A., private communication, 1992. 
[9] Goldmann, H., Uniform Fréchet Algebras, North-Holland Math. Studies 162, North-Holland, Amsterdam, 1990.

[10] Grothendieck, A., Sur les espaces $(F)$ et $(D F)$, Summa Brasil. Math. 3 (1954), 57-122.

[11] Hörmander, L., An Introduction to Complex Analysis in Several Variables, North-Holland Math. Library 7, North-Holland, Amsterdam, 1973.

[12] Komatsu, H., Ultradistributions I. Structure theorems and a characterization, J. Fac. Sci. Univ. Tokyo 20 (1973), 25-105.

[13] Meise, R. and Vogt, D., A characterization of quasi-normable Fréchet spaces, Math. Nachr. 122 (1985), 141-150.

[14] Michael, E., Locally multiplicatively convex topological algebras.

Mem. Amer. Math. Soc. 11 (1952).

[15] Mujica, J., A completeness criterion for inductive limits of Banach spaces. In: Functional Analysis, Holomorphy and Approximation Theory II, edited by G. Zapata, North-Holland Math. Studies 86, North Holland, Amsterdam, 1984, pp. 319-329.

[16] Mujica, J., Complex Analysis in Banach Spaces, North-Holland Math. Studies 120, North-Holland, Amsterdam, 1986.

[17] Ng, K.F., On a theorem of Dixmier, Math. Scand. 29 (1971), 279280 .

[18] Oka, K., Domaines convexes par rapport aux fonctions rationelles, J. Sci. Hiroshima Univ. 6 (1936), 245-255.

[19] Palamodov, V.P., Linear Differential Operators with Constant Coefficients, Die Grundlehren der Mathematisches Wissenschaften 168, Springer, Berlin, 1970.

[20] Petzsche, H.J., Some results of Mittag-Leffler type for vector valued functions and spaces of class A. In: Functional Analysis: Surveys and Recent Results II, edited by K.D. Bierstedt and B. Fuchssteiner, NorthHolland Math. Studies 38, North-Holland, Amsterdam, 1980, pp. 183204. 
[21] Waelbroeck, L., Duality and the injective tensor product, Math. Ann. 163 (1966), 122-126.

\author{
Instituto de Matemática \\ Universidade Estadual de Campinas \\ Caixa Postal 6065 \\ 13081-970 Campinas, SP \\ Brazil
}

Recibido: 3 de Enero de 1994 